\title{
Polysèmes
}

Revue d'études intertextuelles et intermédiales

\section{La Fin de Foe de J.M. Coetzee : dans l'aporie se glisse le lecteur}

Anne Combarnous

\section{(2) OpenEdition}

\section{Journals}

\section{Édition électronique}

URL : http://journals.openedition.org/polysemes/1704

DOI : 10.4000/polysemes.1704

ISSN : 2496-4212

Éditeur

SAIT

\section{Édition imprimée}

Date de publication : 1 janvier 2007

Pagination : 101-114

ISSN : 0999-4203

\section{Référence électronique}

Anne Combarnous, «La Fin de Foe de J.M. Coetzee : dans l'aporie se glisse le lecteur », Polysèmes [En ligne], 8 | 2007, mis en ligne le 01 janvier 2007, consulté le 19 avril 2019. URL : http:// journals.openedition.org/polysemes/1704; DOI : 10.4000/polysemes.1704

Ce document a été généré automatiquement le 19 avril 2019.

Polysèmes 


\title{
La Fin de Foe de J.M. Coetzee : dans l'aporie se glisse le lecteur
}

\author{
Anne Combarnous
}

1 Métafiction résolument postmoderne, récit de type narcissique, $\mathrm{Foe}^{1}$, comme son titre le souligne, offre, entre autres, une relecture des romans de Daniel Foe (devenu Defoe par coquetterie toute sociale, ou peut-être par désir de ne pas livrer sa personne physique en signature de ses écrits), une relecture en particulier de son Robinson Crusoe (1719), à la lumière de The Fortunes and Misfortunes of the Famous Moll Flanders (1722), et de Roxana, or the Fortunate Mistress (1724).

2 Texte hybride, Foe est fait d'une première partie proprement narrative, d'une deuxième mêlant le style épistolaire à celui du journal intime, les lettres n'étant pas matériellement adressées à leur destinataire, d'une troisième essentiellement dialoguée mais dont le cadre est un récit au passé, toutes relatées par une narratrice représentée, et d'une dernière partie dont le narrateur est un «je » irréaliste et irréel. Par son nom, la narratrice des trois premières parties, Susan Barton, n'est pas sans rappeler Roxana (une autre Susan). Chez Coetzee elle est celle qui révèle à Foe, son interlocuteur, l'existence de l'île, de Robinson et de Friday. Le texte se clôt sur une très brève dernière partie, énigmatique, constituée de deux fins, dont la dernière remet en question les textes qui précèdent. Le narrateur représenté n'est vraisemblablement plus Susan, retrouvée morte, enlacée dans les bras d'un autre, mais un narrateur anonyme, à l'instar du « darker author » qu'elle imaginait capable, lui, de raconter l'histoire mystérieuse de Friday, aporie au centre du roman, point de focalisation de son discours. Comme le Robinson de Defoe avant elle, Susan a ramené Friday de l'île (contre son gré), comme elle a voulu ramener Robinson, mort cependant de fièvre pendant le trajet. Et c'est l'histoire de Friday, dont pourtant elle ne peut recouvrer la vérité - celui-ci ayant eu, avant même qu'elle ne le rencontre, la langue coupée - qu'elle pose comme la clé de l'histoire de l'île, qu'elle substitue à sa propre histoire et qu'elle a demandé à l'écrivain Foe de faire revivre pour elle, se sentant incapable de l'écrire, ou d'en assumer, en tant que femme peut-être, l'autorité. 
Ce «darker author» de la dernière partie, «je» non instancié, figure bien sûr, ironiquement, la trace du véritable auteur du roman, Coetzee, qui se plait à dévoiler l'artifice (ce qui paradoxalement n'enlève rien à la force de la fiction), mais pas seulement. C'est aussi la figure de cette place vide que le lecteur est censé venir habiter à la lecture, mais aussi peut-être la figure d'une Susan transfigurée en auteur, et ayant pour ce faire délaissé son identité de sujet, ou encore celle d'un Friday « dark to [Susan's] English eye » enfin maître de l'alphabet que lui auraient enseigné Susan et l'écrivain.

4 Une des problématiques centrales du roman est celle que posent, selon Linda Hutcheon, les «métafictions historiographiques $»^{2}$ : comment rendre, par le biais de la narration, la vérité historique, là est la question, que ne posait d'ailleurs pas Defoe lorsqu'il assurait à ses lecteurs, dans sa préface à Roxana : «the Foundation of This is Laid in Truth of Fact; and so the Work is not a Story but History $»^{3}$. (Certes, Defoe jouait sur un autre type d'illusion romanesque.)

5 Susan se déclare engagée du côté du récit-vérité - et son récit est vérité en ce qu'il met à jour, comme réels ou historiques, les procédés mêmes de fictionalisation du réel. Elle rejette l'idée, suggérée à elle par Foe, d'ajouter à son histoire des éléments qui sont majoritairement en fait ceux de l'intrigue du Robinson Crusoe de Defoe : les cannibales, le sauvetage par Robinson de Friday, etc., des inventions en effet de Defoe, comme son imbrication d'ailleurs des deux histoires d'Alexander Selkirk l'explorateur et de l'Indien Friday (redéfini ici comme Africain, en accord sans doute avec le contexte de production du roman, l'Afrique du Sud en révolte des années quatre-vingt). Et Foe d'apparaître au lecteur comme l'opportuniste que son modèle historique était peut-être en effet, puisqu'il est suggéré qu'il s'est servi de l'idée originale de Susan pour en faire son premier roman à succès, et gagner une postérité quand elle n'atteint pas même la reconnaissance et la délivrance qu'elle attendait, finalement réduite qu'elle est au rang de femme-objet, sans apparaître même dans le roman de l'écrivain ; elle n'« apparaîtra » que dans ses romans ultérieurs, ramenée là, en outre, à l'histoire personnelle qu'elle voulait taire, une histoire seulement évoquée, mais similaire en bien des points, en effet, à celle de Roxana ou de Moll. On le comprend, la lecture des classiques est un prérequis pour le lecteur de Coetzee, qui, dans son parcours d'universitaire, se divertit tout en recréant le sens, ou plus précisément peut-être le pourquoi du récit de Defoe.

6 L'engagement de Susan à respecter la vérité est problématique cependant lui aussi. C'est avant tout un produit fini qu'elle désire, qui signerait sa libération en lui procurant la richesse (car elle sent bien qu'elle tient le bon filon!), en faisant d'elle aussi un véritable personnage, comme ont pu le devenir certains témoins de l'histoire (tel Selkirk, dont le récit de son séjour sur une île déserte fut un best-seller de l'époque). Mais de quelle vérité parle-t-elle dans ses lettres à Foe, elle qui refuse aussi cette proposition que lui fait l'écrivain de relater sa propre histoire, sa propre vérité, celle de la recherche de sa fille, enlevée dans ses jeunes années? C'est cet enlèvement qui l'a poussée à quitter l'Angleterre pour se rendre à Bahia, où elle aura vécu quelques années avant de s'en retourner bredouille, de se retrouver débarquée dans une chaloupe par des mutins en compagnie d'un capitaine assassiné, et d'aller rejoindre à la nage l'île de la Désolation, action sur laquelle s'ouvre le roman. À l'histoire de cette quête, qu'elle refuse de relater autre aporie au cœur du récit -, Susan substitue celle, irracontable, de Friday, à qui sans en avoir conscience elle a retiré la liberté, et dont elle s'est donné, comme d'un enfant, la responsabilité. 
7 À la lumière de cette lecture, c'est bien sûr à la déconstruction-reconstruction du roman de Defoe que le lecteur est invité, comme il est invité à reconsidérer essentiellement le rôle colonisateur de Robinson Crusoe dans la soumission, volontaire (?) de Friday. D'autre part, Susan rejetant son passé de mère, allant jusqu'à ne pas reconnaître la jeune femme qui se présente à elle et dit être sa fille, une seconde Susan Barton, rappelle Roxana qui abandonnait ses enfants pour profiter des biens matériels de nombreux amants qui l'entretenaient tour à tour, ignorants de sa progéniture. Mais contrairement à Roxana, Susan, parce qu'elle s'interroge sur l'exploitation de l'homme par l'homme (les relations de Robinson et de Friday essentiellement, car elle ne prend que graduellement conscience de sa propre exploitation de Friday), se montre davantage encline à rejeter des relations de prostitution dont elle cherche à se délivrer, qu'elles soient passées (telles que l'on peut l'imaginer en avoir entretenu à Bahia, où elle dit avoir été une femme « libre ») ou présentes (elle fait mention des nuits qu'elle passe dans les bras de son vieux logeur, un moyen pour elle d'avoir un toit à bon marché, ce qu'elle n'explicite pas justement, pour mieux s'en délivrer peut-être). En revanche elle est prête à dévoiler ses relations sexuelles avec Cruso, par exemple, qui peuvent davantage figurer comme un don pur, dont elle n'aurait pas à rougir. Ces indices nous font également porter un autre regard sur le Robinson Crusoe de Defoe, sur la mention finale, notamment, des convois de femmes destinés à assurer la continuité de la présence des Européens sur l'île. Il semble bien s'agir dans le roman de Coetzee d'une dénonciation déguisée de l'imbrication inévitable de l'exploitation des femmes (et des hommes) et d'un impérialisme soi-disant humaniste tel qu'il était implicitement prôné par Defoe, dans ces premiers temps de l'essor du commerce de l'Angleterre avec l'Asie, l'Afrique et l'Amérique.

Dans l'histoire de Friday, telle que Susan en relate certains détails présents, c'est en fait celle de Susan que l'on peut lire en filigrane, comme le moyen pour elle de se livrer sans se livrer, en attendant que Foe, tel un analyste dans l'esprit aveuglé de l'analysé, lui offre la délivrance de son passé, lui donne les clés de sa propre histoire. Friday fait figure d'écran et dans sa sujétion, c'est celle de Susan que l'on peut lire aussi, qui nous fait comprendre sa culpabilité de femme facile, soumise au pouvoir économique et juridique des hommes.

9 Finalement, après s'être battue longtemps pour défendre son idée de vérité, Susan renoncera, semble-t-il, à celle-ci, en se rangeant du côté de Foe, tout en croyant cependant l'avoir gagné à son idée. C'est ainsi que j'interprète son acte symbolique de renversement du pouvoir, dans la troisième partie, lorsqu'elle le chevauche dans leur acte d'amour, telle la Muse le poète, dit-elle. En fait, par cet acte, Susan revient à l'ordre de la matière, elle est rapportée à sa "substance " propre, son corps de femme, identité problématique qui la signe comme potentiellement achetable par les hommes. Ainsi disait-elle à Foe en amont :

I could return in every respect to the life of a substantial body, the life you recommend. But such a life is abject. It is the life of a thing. A whore used by men is used as a substantial body. (125-126)

Dans la dernière page de cette même section, elle en vient à s'inclure pourtant parmi les êtres substantiels, de même qu'elle veut bien reconnaître la seconde Susan Barton comme être de substance, et non plus de fiction, omettant de mentionner Friday comme faisant partie de cette chaîne de vie ( We are all alive, we are all substantial, we are all in the same world. " "You have omitted Friday. » 152). Il est significatif qu'à cette omission corresponde le fait bien sûr que c'est lui maintenant qui s'adonne à l'écriture, assis au 
bureau de l'écrivain et traçant voluptueusement sur les pages que lui a laissées celui-ci des lignes de «o", promesses pour Foe de "a " à venir (omega et alpha bibliques inversés, quand la fin rappelle - ou révèle ? - le commencement).

11 Au désir de vérité, et aux atermoiements de Susan sur son incapacité à restituer celle-ci et sur la lenteur de Foe à se mettre à la tâche (reflet de cette "vie de pénitence » en Angleterre à laquelle Moll Flanders se voue à la fin de sa "mauvaise vie», pénitence coïncidant avec l'écriture de cette même vie à laquelle elle vient de s'adonner) fait place le récit onirique de la quatrième et dernière partie, où le "je » se confond d'abord avec celui de Susan, reprenant mot pour mot la toute première phrase de la troisième partie à l'exception du temps toutefois, le présent ici -, pour se révéler autre, découvrant le corps de Susan, non nommée mais reconnaissable, morte dans les bras de Foe, lui-même cadavre, tandis que Friday repose dans l'alcôve sombre de la pièce, survivant lui. N'ayant pas écrit, ayant renoncé à l'écrit, Susan se serait-elle vouée à la mort, aurait-elle voué à la mort son âme - ce que cette mort figurée sur la page symboliserait ? Il y aurait alors, ici, comme chez Defoe, l'idée d'une fonction rédemptrice de l'écrit (confessionnel ou non), ou plutôt une fonction cathartique car il semble inclure dans sa structure l'écrivain luimême, quand Defoe, lui, se posait davantage, il me semble, en détenteur d'une morale inattaquable, dénué donc de tout vice. On pourrait arguer, bien sûr, que par le seul choix de ses sujets féminins, Defoe dévoilait obliquement, sans même en avoir conscience peutêtre, des failles qu'avec elles il aurait pu partager - comme lorsqu'il se vendit en quelque sorte, pour servir le gouvernement Tory de Robert Harley de 1703 à 1714, en échange de sa libération de la prison de Newgate, où il avait été enfermé en 1702 pour avoir attaqué l'église catholique extrémiste.

12 La deuxième fin de Foe (ou la troisième, pourrait-on dire) fait suite à la plongée métaphorique du narrateur obscur dans les eaux du texte précédent de Susan (pré-texte, prétexte), le manuscrit trouvé dans la maison du célèbre écrivain, ici inscrit comme « Daniel Defoe ». Cette plongée, que souligne aussi l'emploi du présent simple dans toute cette section, temps du décrochage fictif et du rêve par excellence, ainsi, paradoxalement, que de la description objective des faits, est marquée par la reprise des premiers mots du roman (« At last I could row no further. [...] With a sigh, making barely a splash, I slipped overboard.» 5), en citation d'abord, puis comme une réappropriation («Bringing the candle nearer, I read the first words of the tall, looping script: 'Dear Mr Foe, At last I could row no further.' With a sigh, making barely a splash, I slip overboard.» 155). Seules les références au corps de Susan ne sont pas reprises («My hands were blistered, my back was burned, my body ached.»5). Ceci n'a rien de surprenant, soit que les mots se perdent dans une lecture plus silencieuse, soit qu'il s'agisse bien ici pour l'écrivain d'évoquer une voix désincarnée. Est ajoutée la référence au destinataire du manuscrit, révélé non au début mais seulement dans la deuxième partie du roman, une manière ici de mettre en avant, pour mieux l'interroger ensuite, la nature du récit - de nous faire remarquer aussi peut-être que c'est seulement dans cette dernière section que le texte n'est pas du " discours ", écrit ou rapporté.

$13 \mathrm{Au}$ fond de ces eaux-là du texte perdu et retrouvé, le narrateur découvre l'épave d'un bateau, et dans celle-ci, le cadavre de Susan à nouveau, gonflé par les eaux, dans les bras cette fois de "son capitaine " (" of her dead captain», une référence qui brouille les pistes, évoquant le capitaine d'un navire dont elle n'aurait pas été chassée dès lors, et qui rendrait l'île comme tout le récit à sa nature fictive, à ce qu'ils sont vraiment, une reconstruction, telle celle effectuée par Defoe, dissimulant peut-être, à l'image de celle de 
Susan, une vérité inavouée parce qu'inavouable). Friday, là encore, se trouve recroquevillé dans un coin de l'épave, mais malgré les mouvements miraculeux de son corps (« He turns and turns till he lies at full length, his face to my face. » 157), il ne paraît plus vivant : il porte au contraire ce même masque de mort que les cadavres enlacés de la première fin («The skin, dry as paper, is stretched tight over their bones. Their lips have receded ", 153, devient: "The skin is tight across his bones, his lips are drawn back. » 157). La vérité historique qui transparaît dans ces images, bien sûr, c'est celle que signalait déjà Friday sur l'île, dans l'acte rituel d'aller jeter sur la mer des pétales de fleurs : celle des milliers de morts laissés au fond des mers, n'ayant pas survécu au voyage qui les promettait sûrement à l'esclavage, la vérité des peuples opprimés noyés dans les eaux du texte dominant de l'Occident, menaçant peut-être de se révéler dans un ultime mouvement de rébellion (Friday, les lèvres rétractées, semble, là, prêt à mordre, comme dans les fantasmes antérieurs de Susan - peur primitive, peur d'une punition non assimilée encore comme punition).

Et cette vérité-là se révèle à nous à la lecture du texte, dans toute son absurdité, sa petitesse - dans tous les sens du terme -, en regard de l'immensité océane et des continents parcourus par le souffle magique, pour ne pas dire « divin » de Friday, souffle sans souffle, qui pourrait être dit désincarné s'il n'était tant montré dans son rapport au corps qu'il parcourt aussi, et s'il ne venait pas nous toucher comme il le fait, droit au cœur. Autre demande, embarrassée, faite aux peuples opprimés : de ne pas répondre au mal par le mal si l'occasion leur en était donnée, mais par une nouvelle énergie qui saurait insuffler au monde le souffle peut-être d'une voix propre, que l'auteur ici ne se réapproprie d'ailleurs pas, et qui saurait se faire entendre.

His mouth opens. From inside him comes a slow stream, without breath, without interruption. It flows up through his body and out upon me; it passes through the cabin, through the wreck; washing the cliffs and shores of the island, it runs northward and southward to the ends of the earth. Soft and cold, dark and unending, it beats against my eyelids, against my face. (157)

Dans ce «darker author » de la troisième partie, il est possible de reconnaître une Susan gagnée à l'idée défendue par Foe de la supériorité de l'imaginaire sur la vérité (sinon sur le réalisme), un Foe, médium finalement d'une certaine vérité, non dans sa morale puritaine, telle qu'elle apparaît dans Robinson Crusoe, Roxana ou Moll Flanders, mais dans le simple fait de re-présenter cette morale - un Foe dépassé en outre par son élève, à moins qu'il n'ait de près participé à l'ébauche finale du nouveau type de récit qui nous est finalement proposé. Susan, enfin réconciliée avec son « darker self », se serait donné les moyens peut-être de livrer au lecteur une image non équivoque, bien qu'impossible de cette vérité - en se re-présentant dans des postures qui pourraient porter atteinte à sa réputation de "femme libre», et comme ayant partie liée avec la mort, ou l'enchaînement de Friday.

Est mise en avant en tout cas l'idée de la nécessaire invention, d'un nécessaire travail de recréation, de la nécessaire métaphore, l'idée d'une écriture autre que mimétique exclusivement, car la vérité ne saurait surgir d'une "garantie » ou d'un "décret » de l'auteur/narrateur, selon les mots de J.P. Audigier". Dans cette fin, qui se donne a lire comme une plongée métaphorique dans l'imaginaire, se lit la révélation d'une vérité imagée, la puissance du non-dit, de la vérité à jamais voilée de la prose poétique. Elle figure l'acceptation d'une mise en ordre symbolique autre, où l'imaginaire, qui dit pourtant l'impossible, aurait également sa place. Elle montre l'écriture non plus comme « expression », mais comme " production », « surgissement », « processus » (comme un 
laisser-aller aussi au plaisir charnel des mots), à l'image du vent qui sourd de la bouche de Friday, lui-même image de ce que Deleuze et Guattari dans L'Anti-Oedipe nomment la «machine productrice» de «flux décodés du désir qui fuient, s'écoulent, et nous entraînent où? " ${ }^{5}$. Il s'agirait ici de "recréation» - au sens classique du terme: " rétablissement ", manière de "faire revivre » le sens de la littérature, si tant est que la littérature puisse être " guérie » - un message prégnant dans le contexte de l'Afrique du Sud d'alors, où la pléthore des romans à thèses, des romans à " messages ", affaiblissait sans doute l'efficacité (même révolutionnaire) du roman. En regard de l'art du roman, pour Coetzee peut-être, comme pour Robbe-Grillet, c'est le travail sur la forme qui peut se révéler révolutionnaire ${ }^{6}$.

La deuxième problématique mise en avant dans Foe est celle d'un paradoxe intrinsèque à la littérature, la difficulté de dire le non-dit (« Till we have spoken the unspoken, we have not come to the heart of the story. » dit Foe, 141), la difficulté de faire parler l'autre, privé de voix, témoin muet de l'histoire, sans le réduire à un statut d'objet. Friday est défini comme « le trou dans le récit» ( " the hole in the narrative ») que Susan voudrait trouver le moyen de remplir, et qui l'aspire, de la même façon que le trou dans sa bouche est la trace d'une histoire qu'elle voudrait recouvrer et qui la fascine ( It is for us to open Friday's mouth and hear what it holds: silence, perhaps, or a roar, like the roar of a seashell held to the ear ", 142). C'est parce qu'il est mutilé que le corps de Friday devient objet de fascination, et fait sens pour elle. Et il est important de noter qu'elle imagine une mutilation plus terrible encore que celle de sa langue coupée, dont elle croit parfois Cruso responsable. Ce que renferme ce corps, c'est le secret de la dépossession de l'homme par l'homme, et quelle dépossession plus terrible que celles des parties génitales reproductrices? Son fantasme ne reflète-t-il pas en fait ses propres angoisses de dépossession, que lui rappellerait son propre " trou », tout féminin celui-là, autre « trou » du récit? Ce qu'elle verra sous la robe de Friday, elle ne le dira pas, mais peut-être est-ce la raison pour laquelle finalement il perdra pour elle de sa substance, comme ayant perdu de cette corporéité émasculée, définie pour lui par les autres, par la loi dont Susan est imprégnée, celle des esclavagistes, des colons envahisseurs, qui sans se priver de rapports occasionnels avec les femmes «de » l'autre, s'arrogent, par compensation d'une faiblesse, sans doute, à cet égard, l'exclusivité des rapports avec les « leurs ». Ce qu'elle a vu, c'est peut-être bien que cette émasculation qu'elle imaginait n'était pas. Est-ce pour cela qu'elle ne le dévoile pas? Ne révélant pas le subterfuge du fantasme inculqué par la loi, elle semble montrer combien, à celle-ci, elle reste soumise en tout cas.

Susan est incapable en réalité de reconnaître Friday comme autrui, la «structure Autrui », comme la dénomme Deleuze dans sa post-face au Vendredi ou les Limbes du Pacifique de Michel Tournier (1969) ${ }^{7}$, ayant là aussi disparu peut-être au profit d'une «structure perverse » que Deleuze donne comme point de départ de la robinsonnade. Ce faisant, cependant, elle se donne peut-être le moyen de découvrir en lui l'« au-delà d'Autrui ", un double, figure réversible d'elle-même en réalité. Mais il reste que Friday, sans choix possible, lui, est soumis.

19 Friday est le signe dans le roman d'un référent masqué, comme lorsque Susan le décrit dans sa transe, tournant sur lui-même, enveloppé de la robe rouge de l'écrivain qui forme un cercle autour de lui. "Dark pillar » au centre d'un carré de soleil, « pilier central », il est signe d'un lien spirituel entre l'être et le monde, d'une religion autre ${ }^{8}$. Il est l'image de celui qui, dépossédé, possède encore, au-delà de la surface de son corps, une âme, l'image de celui qui ne saurait être réduit, comme elle le réduit pourtant, au statut de victime de 
la cruauté. Ce qu'elle ne voit pas c'est qu'il est victime de sa propre perversion, de cette même réduction qu'elle lui impose dans son désir inconscient de se dire elle, peut-être, à travers lui.

Dans sa transe, Friday devient le signe d'une abstraction de soi à soi-même, dans l'unique expression de son corps, de son être de chair, signe non pas d'une transcendance comme celle que recherche Susan à travers les mots (à l'instar du Robinson de Defoe), mais d'une transcendance dans l'immanence. C'est dans cette sortie de soi, cette possession autre de l'esprit par les sens, que Friday semble atteindre l'en-soi, signifiant la véritable histoire peut-être que, lui, pourrait vouloir raconter : celle de sa liberté sur l'île à laquelle Susan l'a arraché, comme semble le rappeler aussi le souffle infime qui sourd de sa bouche dans la première version de fin du roman: "From his mouth issue the sounds of the island" (154). Ce sont ces mêmes bruits, cette fureur du vent de l'île et des cris d'oiseaux justement que Susan, sur l'île, voulait fuir, comme si elle avait désiré fuir sa propre insignifiance, ainsi révélée par tout un univers totalement indépendant de l'homme. Son désir de fuir cette vie-là la poussait à aller s'immerger pour ne plus les entendre, ou à rester prostrée dans un coin de roche (comme le Robinson de Tournier), dans un repli conscient sur son corps propre - opposé en image à ce tournoiement solaire de Friday (qui rappelle le Vendredi aérien de Tournier).

C'est dans l'écrit (repli ?) que Susan veut rendre la vie, elle qui ne sait pas vivre, elle selon qui il aurait existé pour Friday un âge d'or de la parole, d'une parole rêvée en adéquation avec le réel, ignorante qu'elle semble être de la supériorité des sens sur les mots. Et sa quête est absurde bien sûr.

Le renoncement final à l'écrit que semble figurer la dernière partie pourrait cependant signifier sa découverte d'une autre valeur possible des mots : celle qui se fait jour dans les jeux de construction, reconstruction, création, recréation, récréation, appropriation, distanciation de l'écrivain ironiste. C'est elle-même peut-être en effet qui se serait représentée morte dans les bras d'un homme, image de sa compréhension de l'impasse dans laquelle elle s'était enfermée en laissant à Foe la tâche d'assumer pour elle son histoire, en se laissant réduire par lui à un rôle d'objet (de ses désirs comme de l'histoire). Il s'agirait aussi d'une sorte d'expiation symbolique de son crime, celui d'avoir arraché Friday à son île. La chaîne qu'il a autour du cou dans cette dernière partie (en lieu et place de la pochette contenant la lettre de manumission qu'elle avait écrite pour lui) pourrait bien figurer en effet la chaîne de mots dans laquelle elle voulait l'enfermer.

Mais la fin de l'écrit pourrait aussi signaler le début de la lecture, et ce qui se trouve représenté ici, de manière imagée, ne serait rien d'autre que la « substantifique moelle » des récits et discours antérieurs de Susan.

Par cette image onirique du souffle sans souffle, de cette voix sans voix qui sourd de la bouche de Friday, figure d'une autre vie possible, Coetzee révèle comme il le résout, en partie, ce paradoxe intrinsèque à la littérature qui est de dire le non-dit, l'indicible, auquel renvoient la figure énigmatique de Friday, les lignes de «o » tracées par lui, le « $\mathrm{O}$ » que dessine la bouche de la seconde Susan Barton, voire le cercle central du O de Foe sur la couverture du roman dans l'édition Penguin.

La fin fonctionne comme une résolution de cette lutte acharnée de Susan contre le bruyant silence existant hors des mots, le silence oppressant de Friday, «fumée noire » asphyxiante (après le silence tout aussi oppressant d'un Cruso peu bavard). Mais si son manque d'art (ou d'«artifice ») à raconter l'histoire, son manque de maitrise du langage 
(impérialiste ?) la contraignait à subir ce silence, c'est lui encore qui domine dans l'image saisissante de la fin, pirouette encore en ce qu'elle est une figuration possible du silence des mots mêmes dès lors qu'ils sont écrits sur la page et frappent l'attention du lecteur comme figures de ce même indicible : « Soft and cold, dark and unending, it beats against my eyelids, against the skin of my face. » (157).

À l'image $d u$ «o », cercle vide, mais lettre la plus «parlante » peut-être de l'alphabet (comme le «a»), tel le « 0 » du Friday de Defoe, interprété par Robinson comme une prière au Dieu auquel Friday, suivant son maître, se serait converti, la fin du roman matérialise le silence signifiant de Friday, figurant ainsi peut-être la nécessaire insubstantialité du sujet écrivant; le "je » qui énonce a perdu sa valeur de référence, s'offrant comme un espace habitable par le lecteur, comme par ces voix silencieuses, " contrevoix » par lesquelles Coetzee se dit être habité dans l'espace de l'écriture', et qui comprennent peut-être la voix de certains personnages de ses lectures antérieures. C'est cette récréation-ci qui se trouve révélée, rétablissement d'une partie comme clivée du moi, détentrice d'une certaine "vérité », qui ne saurait surgir que dans les mots d'un sujet effacé. Le lecteur à l'image du narrateur énigmatique est invité à tendre, comme l'écrivain, l'oreille, à se couper le souffle pour mieux être à l'écoute, comme accepter de ne plus vivre un temps pour mieux vivre à la fin peut-être. Ce narrateur obscur, c'est un peu comme l'auteur/lecteur idéal qui se tait et écrit, ou lit, les yeux fermés, ouvert à une vision plus intérieure, se laissant engloutir par la goule marine et, ce faisant, découvrant l'horreur (et sa mystique ?) qu'il révèle et rend à la mémoire des hommes comme à la sienne.

Le souffle de Friday figurerait la voix d'un inconscient trop longtemps bâillonné comme le Malin Génie de Descartes - il figurerait le corps parlant à travers l'écrit, seul capable de réveiller la magie du langage, de renouer avec le présent éternel du temps, et seul capable de rappeler le sens perdu du toucher et d'établir ce lien illisible avec le "divin », non corrompu, qu'Artaud évoquait dans sa vision d'un théâtre total et vivant ${ }^{10}$.

Est figurée dans cette section la percée du langage, telle que l'évoquait Barthes ou Blanchot, libérant la littérature de "la loi du signifiant castratrice ${ }^{11}$, ou plutôt manifestant le désir d'en faire autant. Ainsi l'épave de la fin devient, de manière compréhensible (presque), l'endroit où "les corps sont signes d'eux-mêmes", la "demeure de Friday », une façon pour l'écrivain de mettre en avant le corps en tant que signe concret parlant. De même dans la transe, préfiguration du chant final de Friday, il n'y avait plus d'esprit autrement qu'incarné, plus de transcendance autrement qu'immanente, le plaisir de la fuite quand l'être-là n'est plus là, mais fait place à l'étant peut-être - comme l'extase (ek-stase) situe l'illuminé dans le monde et hors du monde, lui fait accéder à l'en-soi « hors de soi ».

29 C'est l'image qui est ressuscitée, avec des mots qui disent l'étonnement premier sur le monde sans l'expliquer. En même temps, s'opère un recentrage sur le rôle du lecteur. L'auteur obscur de la dernière partie, entraînant celui-ci à sa suite à la découverte de ce mystère indicible que dit, sans dire, le texte de Susan (le corps de Friday l'ayant envahi), offre une métaphore de ce que le lecteur, "machine désirante» et "machine productrice ", aura pu lui-même vivre, ou pourra plus précisément vivre à la relecture du texte, en «fais[ant s]iens les mots qu'un autre a pensés pour [lui]», comme l'écrivait G. Poulet ${ }^{12}$.

C'est à la redécouverte d'un corps faisant partie de la chaîne des corps, auquel le texte nous relie, tissu marin, algue qui nous retient, que nous sommes conviés, dans une 
relation autre qu'anthropophage, à une ouverture de notre propre corps peut-être au monde, de notre vision sur le monde. Dans cette affirmation du corps et d'une voix autre, d'autant plus libérée du joug du langage qui emprisonnait Susan que cette voix ne s'attache à aucune réalité de voix, qu'elle est voix hors le sujet, voix hors les mots (morts ?), voix d'une histoire, de l'histoire qui se dit à travers un corps qui parle de luimême, ce dont il s'agit c'est tout bonnement de « donner à voir » (les maîtres mots de Godard, comme de Coetzee).

31 Les mots ramènent toujours à l'idée de surface, celle du montré (comme le corps de Friday est un voile de son âme rentrée). Ce texte surface qui nous est donné, invitant le lecteur à en exhumer le sens, la profondeur cachée, le prive aussi de ce plaisir, en semblant par la vérité imagée, l'image du rêve, tout dire et tout montrer, rendre sa transparence à l'opacité du réel figuré. La question dès lors qui nous est posée, ne serait plus tant liée à notre habileté à révéler, d'un texte, le sens caché, que de savoir quel sens donner à notre activité de lecteur, de livres comme du monde.

Coetzee propose bien ici une relecture démythifiante du Robinson Crusoe de Defoe, qui montre combien ses thèmes ont conservé leur fraîcheur, mais qui permet, étant donné le rôle de modèle généralement attribué à ce texte, de reposer la question du roman, la question de l'usage des mots, du langage, de la langue, et qui se rapproche de la relecture qu'en a fait Tournier, dont le Robinson aérien et solaire, suivant la leçon de Friday, renonce à quitter l'île. Coetzee, cependant, ne semble pas privilégier l'utopie à la manière de l'écrivain français. Signalant au contraire, par ce «non-lieu » que représente sa dernière partie, le caractère utopique de son récit (ou plutôt dystopique, si l'on considère la ruine du texte que symbolise l'épave), il nous rappelle la force des images de nos rêves, et de nos cauchemars, la force des images brutes de l'histoire, et la nécessité peut-être de se recréer un regard, capable, lui, de lire l'opaque, de se faire voyant de ce qui, au fond, n'est que trop visible, mais ne se voit plus, plutôt que de ramener les choses toujours à l'inexplicable (comme le fait Susan, comme moi-même peut-être je l'ai fait) par un désir pervers de tout expliquer, qui ne fait que retarder le ressentir, et l'agir (même quand l'agir n'est qu'un « écrire »).

\section{NOTES}

1. Édition de référence : Foe (1986), Harmondsworth: Penguin Books, 1987.

2. Linda Hutcheon, A Poetics of Postmodernism. History, Theory, Fiction, New York \& London: Routledge, 1988.

3. Daniel Defoe, Roxana (1724), Oxford: Oxford UP, 1983, 1.

4. Cf. J.P. Audigier, «Pratiques énonciatives dans le roman (anglais du dix-huitième siècle) : la leçon de lecture ", in Théorie, littérature, enseignement, $\mathrm{n}^{\circ}$ 6, 1988, 69-76.

5. Gilles Deleuze et Félix Guattari, L'Anti-CEdipe. Capitalisme et schizophrénie (1972-73), Paris : Éditions de Minuit, 1992, 161.

6. Alain Robbe-Grillet, Pour un nouveau roman, Paris : Éditions de Minuit, 1961, 39.

7. Également publiée dans La Logique du Sens (1969), Paris: Éditions Minuit/Critique, 1994, 350-372. 
8. Cf. Mircea Eliade, "Symbolisme du 'Centre'», Images et Symboles (1952), Paris: Gallimard, coll. « Tel », 1980, 49-50.

9. Cf. David Attweel, ed., Doubling the Point: Essays and Interviews, Cambridge, Mass.: Harvard UP, 1992, 65.

10. A. Artaud, Le Théâtre et son double (1938), Paris : Gallimard, coll. « Essais », 1964. Voir la préface ( Le théâtre et la culture »), ainsi que "Sur le théâtre balinais » et " Théâtre oriental et théâtre occidental ». Voir également «La Parole soufflée» in L'Écriture et la différence de Jacques Derrida (1967), Paris : Seuil, coll. « Points », 1994, 281-284.

11. Gilles Deleuze et Félix Guattari, L'Anti-CEdipe, op. cit., 261

12. G. Poulet, «Phénoménologie de la lecture » (1969). Cité par Robert Con Davis in Contemporary Literary Criticism. Modernism Through Post-Structuralism, New York \& London: Longman/English Humanities, 1986, 350-362.

INDEX

oeuvrecitee Foe 